\title{
A PROPERTY OF PERMUTATION GROUPS ANALOGOUS TO MULTIPLE TRANSITIVITY.
}

By PROFessor Walter B. CARver and Mrs. estella Fisher king.

(Read before the American Mathematical Society December 31, 1919.)

THE binary forms

and

$$
a_{0} z_{1}^{n}+a_{1} z_{1}^{n-1} z_{2}+a_{2} z_{1}^{n-2} z_{2}^{2}+\cdots+a_{n} z_{2}{ }^{n}
$$

$$
b_{0} z_{1}^{n}+b_{1} z_{1}^{n-1} z_{2}+b_{2} z_{1}^{n-2} z_{2}{ }^{2}+\cdots+b_{n} z_{2}^{n}
$$

are said to be apolar* if

(1) $\quad a_{0} b_{n}-\left(\begin{array}{l}n \\ 1\end{array}\right) a_{1} b_{n-1}+\left(\begin{array}{l}n \\ 2\end{array}\right) a_{2} b_{n-2}+\cdots+(-1)^{n} a_{n} b_{0}=0$.

Let us assume that $a_{0} \neq 0$ and $b_{0} \neq 0$; and let the sets of roots of the two corresponding non-homogeneoust equations

and

$$
a_{0} z^{n}+a_{1} z^{n-1}+\cdots+a_{n}=0
$$

$$
b_{0} z^{n}+b_{1} z^{n-1}+\cdots+b_{n}=0
$$

be respectively $x_{1}, x_{2}, \cdots, x_{n}$ and $y_{1}, y_{2}, \cdots, y_{n}$. Then the condition for apolarity may be expressed in terms of these roots as follows:

$$
\begin{aligned}
\mathrm{S}_{n}-\left(\begin{array}{l}
n \\
1
\end{array}\right)^{-1} \mathrm{~S}_{n-1} \Sigma_{1} & +\left(\begin{array}{l}
n \\
2
\end{array}\right)^{-1} \mathrm{~S}_{n-2} \Sigma_{2}+\cdots \\
& +(-1)^{n-1}\left(\begin{array}{l}
n \\
1
\end{array}\right)^{-1} \mathrm{~S}_{1} \Sigma_{n-1}+(-1)^{n} \Sigma_{n}=0,
\end{aligned}
$$

where the S's and $\Sigma$ 's are the usual symmetric functions of the $y$ 's and $x$ 's respe'ctively.

If now we write the product of the $n$ differences

$$
\left(x_{1}-y_{1}\right)\left(x_{2}-y_{2}\right) \cdots\left(x_{n}-y_{n}\right) \text {, }
$$

permute the $x$ 's in all possible ways (keeping the $y$ 's fixed), and sum the $n !$ products thus obtained, we get a result which is the same as the left-hand member of (2) except for the

* Cf. Grace and Young, Algebra of Invariants, p. 213; or Dickson, Algebraic Invariants, p. 78 .

$\dagger$ This change to non-homogeneous form is only for convenience of expression, and is not essential to the argument. 
factor $(-1)^{n} n$ ! Thus, for $n=3$, the condition for apolarity may be written

$$
\begin{aligned}
& \left(x_{1}-y_{1}\right)\left(x_{2}-y_{2}\right)\left(x_{3}-y_{3}\right)+\left(x_{2}-y_{1}\right)\left(x_{3}-y_{2}\right)\left(x_{1}-y_{3}\right) \\
& +\left(x_{3}-y_{1}\right)\left(x_{1}-y_{2}\right)\left(x_{2}-y_{3}\right)+\left(x_{1}-y_{1}\right)\left(x_{3}-y_{2}\right)\left(x_{2}-y_{3}\right) \\
& +\left(x_{3}-y_{1}\right)\left(x_{2}-y_{2}\right)\left(x_{1}-y_{3}\right)+\left(x_{2}-y_{1}\right)\left(x_{1}-y_{2}\right)\left(x_{3}-y_{3}\right) \\
& \quad \equiv 6 x_{1} x_{2} x_{3}-2\left(x_{2} x_{3}+x_{3} x_{1}+x_{1} x_{2}\right)\left(y_{1}+y_{2}+y_{3}\right) \\
& \quad+2\left(x_{1}+x_{2}+x_{3}\right)\left(y_{2} y_{3}+y_{3} y_{1}+y_{1} y_{2}\right)-6 y_{1} y_{2} y_{3}=0 .
\end{aligned}
$$

But if instead of using all of the six permutations of the $x$ 's, we use only the first three, we obtain exactly the same result except for the numerical factor 2 ; i.e., we may obtain the apolarity condition by using only the alternating group of permutations of the $x$ 's instead of the entire symmetric group. The same is true in the case $n=4$. But for $n=5$ we find that the result may be obtained by using only 10 permutations* which do not form a group at all.

Two questions immediately present themselves; namely, (1) what is the smallest set of permutations which will give the apolarity condition for a given integer $n$; and (2) what is the smallest group of permutations which may be used for the purpose. Our present interest is in a property of permutation groups which is suggested when one attempts to answer the second question. $\dagger$

Definition.-A permutation group will be said to be doubly pseudo-transitive if, for some one pair of marks $x_{m} x_{n}$, there is, for any arbitrarily chosen pair of marks $x_{i} x_{j}$, at least one permutation of the group sending the pair $x_{m} x_{n}$ into the pair $x_{i} x_{j}$ in some order.

In ordinary double transitivity there would have to be one permutation sending $x_{m}$ into $x_{i}$ and $x_{n}$ into $x_{j}$, and another sending $x_{m}$ into $x_{j}$ and $x_{n}$ into $x_{i}$; while in double pseudotransitivity it is only necessary that there be a permutation sending the pair $x_{m} x_{n}$ in some order into the pair $x_{i} x_{j}$.

${ }^{*}$ They are the permutations I, (12345), (12345) ${ }^{2},(12345)^{3},(12345)^{4}$, (2453), (4351), (4125), (5231), and (1342); which are 10 of the permutations of the group of order 20 on 5 marks.

$\dagger$ As to the first question, it may be said in passing that the smallest number of permutations that can be used must obviously be a common multiple of the binomial coefficients for $n$, and it is possibly the least common multiple. So far as the authors are aware, the question has never been fully answered. 
Definition.-A permutation group on $n$ marks will be said to be $k$-ply pseudo-transitive (where $1 \leqq k \leqq n-1$ )* if, for some one set of $k$ marks, there is, for any arbitrarily chosen set of $k$ marks, at least one permutation of the group sending the one set into the other in some order.

It is evident that there is no difference between simple pseudo-transitivity $(k=1)$ and ordinary simple transitivity; and that if a group is $k$-ply transitive it is also $k$-ply pseudotransitive, but not conversely.

Theorem 1. If a permutation group of degree $n$ is $k$-ply pseudo-transitive, it is also $(n-k)$-ply pseudo-transitive.

Theorem 2. An intransitive group can not be pseudotransitive.

In an intransitive group of degree $n$ it is always possible to separate the $n$ marks into two sets such that no mark of either set is sent into any mark of the other set by any permutation of the group. Then the group could not be $k$-ply pseudotransitive for $k \leqq(n / 2)$. For one could make a selection of $k$ marks all from one set, and another selection containing at least one mark from the other set, and no permutation of the group would send the one selection into the other. But if a group can not be $k$-ply pseudo-transitive for $k \leqq(n / 2)$, it can not, by Theorem 1, be $k$-ply pseudo-transitive for any value of $k$.

It may be noted that ordinary transitivity of multiplicity $k$ implies ordinary transitivity of multiplicity $1,2, \cdots, k-1$, and pseudo-transitivity of multiplicity $1,2, \cdots, k, n-k$, $n-k+1, \cdots, n-1$; while pseudo-transitivity of multiplicity $k$ implies only pseudo-transitivity of multiplicity $n-k$, 1, $n-1$, and ordinary simple transitivity.

CoRoLlarry. The symmetric and alternating groups on $n$ marks are k-ply pseudo-transitive for all values of $k$ from 1 up to $n-1 . \dagger$

This is an immediate consequence of the fact that these groups have ordinary transitivity of multiplicity $n$ and $n-2$ respectively.

TheOREM 3. If a permutation group of degree $n$ is $k$-ply pseudo-transitive, its order $m$ is divisible by $\left(\begin{array}{l}n \\ k\end{array}\right)$; and any arbitrary selection of $k$ marks will be sent into any other

* It is convenient to exclude the case $k=n$, as every group would be $n$-ply pseudo-transitive if this were included.

$\dagger \mathrm{A}$ trivial exception is the alternating group of degree 2. 
arbitrary selection of $k$ marks by exactly $m /\left(\begin{array}{l}n \\ k\end{array}\right)$ permutations of the group.

This theorem, as also its proof, is analogous to the similar theorem for ordinary transitivity.

THEOREM 4. An imprimitive group can not be k-ply pseudotransitive for $k>1$.

The proof of this theorem is very similar to that of Theorem 2.

An examination of the primitive groups of degree not greater than 9 shows the following six cases of groups having pseudotransitivity of a multiplicity other than that immediately implied by their ordinary transitivity:

$\begin{array}{cccc}\text { Degree } & \text { Order } & \text { Transitivity } & \text { Pseudo-transitivity } \\ 7 & 21 & \text { Simple } & \text { Double } \\ 8 & 56 & \text { Double } & \text { 3-ple } \\ 8 & 168 & \text { "6 } & \text { "6 } \\ 8 & 168 & \text { "6 } & 4-\text { ple } \\ 9 & 504 & 3-\text { ple } & \text { 6" }\end{array}$

To return to the condition for apolarity, it is evident, from theorem 3, that a necessary and sufficient condition that a group of permutations on the $x$ 's should give this apolarity condition is that the group should be $k$-ply pseudo-transitive for all values of $k$ from 1 to $n-1$. Thus the alternating group will always serve. The smallest group that will answer the purpose for values of $n$ up to 9 is as follows:

$$
\begin{aligned}
& \begin{array}{llllllll}
n=2 & 3 & 4 & 5 & 6 & 7 & 8 & 9
\end{array}
\end{aligned}
$$

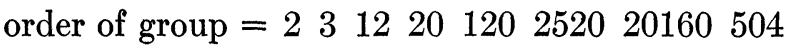

The group is smaller than the alternating group in the cases $n=5,6$, and 9 .

ITHACA, N. Y. 